\title{
Performance Evaluation of PETbox: A Low Cost Bench Top Preclinical PET Scanner
}

\author{
Hui Zhang, ${ }^{1,3}$ Qinan Bao, ${ }^{1}$ Nam T. Vu, ${ }^{1}$ Robert W. Silverman, ${ }^{1}$ Richard Taschereau, ${ }^{1}$ \\ Brittany N. Berry-Pusey, ${ }^{1}$ Ali Douraghy, ${ }^{1}$ Fernando R. Rannou, ${ }^{2}$ David B. Stout, ${ }^{1}$ \\ Arion F. Chatziioannou ${ }^{1}$ \\ ${ }^{1}$ Department of Medical and Molecular Pharmacology, Crump Institute for Molecular Imaging, David Geffen School of Medicine, UCLA, \\ Los Angeles, CA, USA \\ ${ }^{2}$ Departmento de Ingenieria Informatica, Universidad de Santiago de Chile (USACH), Santiago, Chile \\ ${ }^{3}$ Department of Biomedical Engineering, Tsinghua University, Beijing, China
}

\begin{abstract}
Purpose: PETbox is a low cost bench top preclinical PET scanner dedicated to pharmacokinetic and pharmacodynamic mouse studies. A prototype system was developed at our institute, and this manuscript characterizes the performance of the prototype system.

Procedures: The PETbox detector consists of a $20 \times 44$ bismuth germanate crystal array with a thickness of $5 \mathrm{~mm}$ and cross-section size of $2.05 \times 2.05 \mathrm{~mm}$. Two such detectors are placed facing each other at a spacing of $5 \mathrm{~cm}$, forming a dual-head geometry optimized for imaging mice. The detectors are kept stationary during the scan, making PETbox a limited angle tomography system. 3D images are reconstructed using a maximum likelihood and expectation maximization (ML-EM) method. The performance of the prototype system was characterized based on a modified set of the NEMA NU 4-2008 standards.

Results: In-plane image spatial resolution was measured to be an average of $1.53 \mathrm{~mm}$ full width at half maximum for coronal images and $2.65 \mathrm{~mm}$ for the anterior-posterior direction. The volumetric reconstructed resolution was below $8 \mathrm{~mm}^{3}$ at most locations in the field of view (FOV). The sensitivity, scatter fraction, and noise equivalent count rate (NECR) were measured for different energy windows. With an energy window of $150-650 \mathrm{keV}$ and a timing window of 20 ns optimized for mouse imaging, the peak absolute sensitivity was $3.99 \%$ at the center of FOV and a peak NECR of $20 \mathrm{kcps}$ was achieved for a total activity of $3.2 \mathrm{MBq}(86.8 \mu \mathrm{Ci})$. Phantom and in vivo imaging studies were performed and demonstrated the utility of the system at low activity levels. The quantitation capabilities of the system were also characterized showing that despite the limited angle tomography, reasonably good quantification accuracy was achieved over a large dynamic range of activity levels.

Conclusions: The presented results demonstrate the potential of this new tomograph for small animal imaging.
\end{abstract}

Key Words: Positron emission tomography, PET, Small animal imaging, Performance evaluation

\section{Introduction}

Dositron emission tomography (PET) is recognized as a valuable tool for non-invasive, in vivo imaging of 
biological processes at the molecular level and has found wide applications in pharmacology, genetic, and pathology investigations $[1,2]$. Small laboratory animals are commonly used in these studies, which has led to tremendous efforts in developing prototype small animal PET scanners since the 1990s [3-17], some of which have also been commercialized [18-23] and have been widely used in preclinical research. Commercial small animal PET scanners are generally designed to provide a relatively large field of view (FOV) in both axial and transverse directions in order to cover a wide range of animal species from mice to primates. Scintillator arrays made up of finely segmented discrete crystals are used to achieve high intrinsic spatial resolution, and they are coupled to photomultiplier tubes (PMTs) to form detector blocks. Detector blocks are typically arranged in a polygonal (cylindrical) ring with multiple rings assembled continuously, forming long cylindrical geometries. Hundreds of detector blocks with tens of thousands of crystal elements are required in such configurations [23], making the scanners expensive to manufacture. Issues of system complexity and cost versus performance have been previously addressed by using partial ring or dual-head geometries with a rotating gantry or rotating animal bed [6, 2427]. Despite that, considering the rapid growth in the number of small animal imaging studies, there is still a lack of a range of solutions to accommodate the diversity of preclinical imaging studies that are performed with PET.

Spatial resolution and sensitivity are the two major performance metrics for a small animal PET scanner [28]. Over the last decade, most research efforts have been concentrated on improving system performance to achieve higher sensitivity and image resolution $[29,30]$. While it is always desirable to enhance the performance of an imaging system, for applications such as biodistribution and organ uptake quantification, system performance in terms of spatial resolution is less critical considering the sizes of major organs normally involved in dosimetry calculations [31]. Trade-offs can be made to optimize the imaging system for the specified applications at a reduced cost and complexity. It is also worthwhile to note that genetically modified mice are largely the model of choice in biological research and they represent more than $90 \%$ of the preclinical disease models [32]. Therefore, it is desirable to design and build a scanner specifically for imaging mice, which would also lead to an optimized system design with lower cost. Combining these considerations, a dedicated low cost preclinical PET scanner, referred to as PETbox, is currently under development at our institute, which we believe will be of great interest to the research community.

The PETbox is designed as an integrated and easy to use imaging platform optimized for biologists to perform pharmacokinetic and pharmacodynamic mouse studies. The goal of the design is to obtain a good overall system performance at a reasonably low cost and complexity. Our main performance metrics were to achieve $>3 \%$ peak absolute sensitivity at the scanner center and $\sim 2 \mathrm{~mm}$ image resolution in the reconstructed coronal planes. These combined metrics for resolution and sensitivity are adequate for typical biodistribution and major organ level uptake quantification studies [33] and are comparable to the performance of conventional full-ring tomographic systems [34]. Imaging throughput is another important issue for these kinds of studies because it is often necessary to image a considerable number of subjects in a study [35]. This is addressed by optimizing the system geometry for the highest sensitivity achievable, thus maintaining the required scan time, and more importantly, through the integration of a complete animal management system to facilitate animal preparation and handling during the study. It is not uncommon that animal preparation takes an amount of time at least equal to the actual imaging time [36]. It was also shown that the level of radioactivity currently administered during typical preclinical PET imaging procedures leads to a radiation dose for which radiobiological effects have been reported $[37,38]$. PETbox is specifically designed to work at a much lower radioactivity concentration in order to reduce the dose delivered to mice, especially in the case of longitudinal studies involving multiple experiments. For typical PETbox biodistribution studies, we anticipate an administered radioactivity amount of $1.85 \mathrm{MBq}(50 \mu \mathrm{Ci})$, which is a factor of four times less compared to established procedures.

Monte Carlo simulations have been performed on the design of the PETbox scanner to guide decisions on major system parameters and provide quantitative analysis of system performance. That work has been included in a different manuscript submitted separately [39]. Based on the simulation results, a prototype system has been developed and preliminary results of the system were reported in [40]. Main components of this prototype system such as the detector module and system electronics are described in [41, 42]. In this paper, we report on the performance parameters of the integrated system based on the NEMA NU 4-2008 standards [43], modified as necessary to accommodate its non-standard geometry. We also present phantom and in vivo animal studies to demonstrate the capabilities of this new tomograph for small animal imaging.

\section{Materials and Methods}

\section{PETbox System Description}

Fig. 1a shows a schematic drawing of the PETbox scanner. A bench top configuration is used which also integrates a complete animal management system to provide life support functions, including reproducible animal positioning, temperature control, anesthesia, real-time monitoring of animal respiration, and a pathogen barrier, with the emphasis on ease of use to allow non-imaging scientists to access this technology. The scanner employs compact dual-head geometry similar to those of positron emission mammography (PEM) systems [44] or a high-sensitivity small-animal PET system based on High-Resolution Research Tomograph detector technology [45]. Two flat-panel type detector heads are placed 

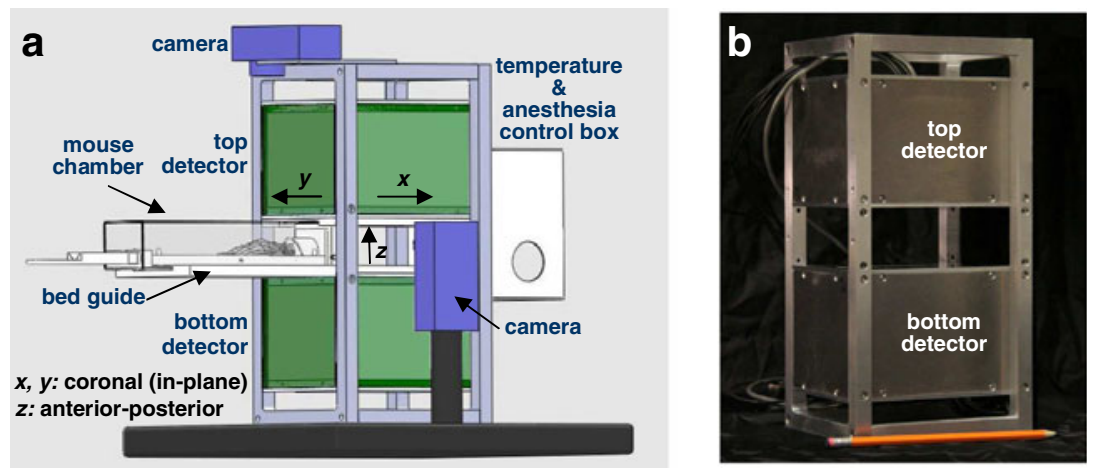

Fig. 1. a Schematic illustration of the PETbox scanner in a bench top configuration. $\mathbf{b}$ A photograph of the gantry with the two detector heads assembled.

opposing each other at a spacing of $5 \mathrm{~cm}$. Each detector has a sensitive area of approximately $5 \times 10 \mathrm{~cm}$, large enough to cover the whole body of the vast majority of laboratory mice $(18-40 \mathrm{~g})$. Mechanical motions of the gantry and the animal bed are avoided in this design in order to reduce system complexity and lower system costs. The two detectors and the imaging subject (mouse) are kept stationary during the scan, providing a limited angle tomography along the central anteriorposterior (AP) view.

PETbox Detectors The design and characterization of bismuth germanate (BGO)-based PETbox detector modules were described by Zhang et al. [41]. The BGO scintillator is used to take advantage of its high stopping power, high photoelectric event fraction and low cost. Additionally, BGO has very low intrinsic radioactivity compared with lutetium oxyorthosilicate (LSO). The significance of this is that system background will not adversely affect the system minimum detectable activity [46, 47], especially considering the very compact geometry of PETbox and the intended use of the system at low activity levels. Each PETbox detector consists of a $20 \times 44$ pixilated $\mathrm{BGO}$ array with a thickness of $5 \mathrm{~mm}$. The individual crystal element in the array has a cross-section size of $2 \times$ $2 \mathrm{~mm}$ and a pitch size of $2.2 \mathrm{~mm}$. The BGO array is coupled to two H8500 multi-channel position-sensitive photomultiplier tubes (PSPMT; Hamamatsu Photonics, Bridgewater, NJ) for scintillation light detection. A charge division resistor network is used to convert the anode outputs from the two PSPMTs into position encoding signals, while the timing signal is obtained directly from the 12th dynode signal on the H8500 PSPMT. Each detector module and the associated readout circuitry is encased in aluminum housing and mounted on a static frame. A photograph of the finished gantry with the two detector heads assembled is shown in Fig. 1 b.

Data Acquisition Electronics Position and timing signals from the two detector modules are directly connected to a field programmable gate array (FPGA)-based fully digital signal processing system which is described in detail in [42]. These signals are digitized and processed using an FPGA device. Event processing algorithms specific to the scanner were implemented in the FPGA device and raw list-mode data containing event information are saved to an external hard disk. A custom graphic user interface was developed for the data acquisition, with which the user can set event detection parameters such as coincidence timing window, and choose the operation mode of the system to collect either singles or coincidence events from the scanner. A delayed window method was also implemented in the FPGA to collect random coincidence events.

Data Corrections and Image Reconstruction The PETbox scanner is currently designed to collect data above a global electronic energy threshold. Energy windowing is applied to the acquired list-mode data afterwards, during data histogramming. Typically the energy window is set to $150-650 \mathrm{keV}$ to allow for higher system sensitivity. For each coincidence event that is within the energy window, the crystal pair is identified and the count for the corresponding line of response (LOR) is increased by one. The randoms are obtained from a delayed window method, and they are subtracted in a similar fashion during histogramming. Normalization is applied on the histogrammed data to correct for the systematic nonuniformities in the detection efficiency of each LOR. These nonuniformities are composed by two parts: (a) system geometry component that comes from detection geometry, crystal penetration, and scatter and (b) variation in detector efficiency due to crystal cutting, light collection, and PMT non-uniformities. The geometry and crystal penetration and scatter component is modeled in a Monte Carlo-generated system probability matrix [48], and therefore, is corrected during image reconstruction. The second component, variation in intrinsic detector efficiency, is corrected by multiplying the measured LOR count with the corresponding normalization factor for that LOR. Since this variation in intrinsic detector efficiency is specific to the components used for the imaging instrument, it only needs to be characterized for one time after the system is built. These normalization factors are obtained from the scan of a uniform plane source. The uniform plane source, which has a total activity of $1.11 \mathrm{MBq}(30 \mu \mathrm{Ci})$, was imaged for $80 \mathrm{~h}$ to ensure adequate statistics $(\sim 3,000$ counts on average per LOR) for generating the normalization factor for each LOR. Attenuation correction is another important data correction for quantitative mouse studies. A method of combining two orthogonal optical views to create a $3 \mathrm{D}$ estimate of the subject was proposed for this purpose [49]. At the time of writing, this method is still under development and optimization, and the results presented here are without attenuation correction (except for the measurement of the image quality phantom). Scatter correction was also not implemented and therefore not included in these results.

An iterative reconstruction method based on a maximum likelihood and expectation maximization (ML-EM) algorithm with the incorporation of a system probability matrix (P-matrix) has been developed to reconstruct 3D images from the limited angle projection data acquired on PETbox [48]. The probability matrix was generated by Monte Carlo-based GATE simulations, and symmetries of the 
PETbox system were utilized to reduce the computation time and increase the counting statistics. The sensitivity of each plane was precalculated to scale the simulation time, which ensured that each element in the probability matrix received about the same amount of counts and that the P-matrix Poisson noise contribution to signal-tonoise ratio is uniform. For a typical study, 30 iterations are used for the reconstruction of image data. The reconstructed image matrix size is $80 \times 80 \times 176$ with a cubic voxel of $0.55 \mathrm{~mm}$ edge length. The entire reconstructed FOV is $44 \times 44 \times 96.8 \mathrm{~mm}^{3}$.

\section{Image Spatial Resolution}

The image spatial resolution of the PETbox system was measured using a $750 \mathrm{kBq}(20.3 \mu \mathrm{Ci})$ NEMA NU4-2008 type ${ }^{22} \mathrm{Na}$ point source with a nominal size of $0.3 \mathrm{~mm}$, embedded in a acrylic plastic cube measuring $10 \mathrm{~mm}$ on all sides (Isotope Products Laboratories, Valencia, CA). The source was attached to a translation stage and positioned between the two detectors. Starting from the center of the FOV, the source was stepped across the central coronal plane towards its edges in both the $x$ and $y$ directions. The step size was $10 \mathrm{~mm}$ along the $x$ direction and $5 \mathrm{~mm}$ along the $y$ direction. Listmode data were acquired at 20 positions in this coronal plane. The source was then raised by $6 \mathrm{~mm}$ along the $z$ direction and stepped across that offset coronal plane in the same manner. For all the acquisitions, the timing window was set to $20 \mathrm{~ns}$.

The list-mode data acquired at each location were histogrammed with an energy window of 150-650 keV. Randoms were corrected before the images were reconstructed using the ML-EM algorithm. The response function was formed by summing $1 \mathrm{D}$ profiles that were parallel to the coronal $x$ and $y$ directions and the $z$ direction (AP direction) and within two times the FWHM of the orthogonal directions. As specified in the NEMA NU-4 2008 protocol, a parabolic fit of the peak point and its two nearest neighboring points were used to determine the maximum value of the response function. Linear interpolation between adjacent pixels was used to determine the position of the half and the one tenth of the maximum; the FWHM and full width at tenth maximum were determined for each extracted profile. The volumetric spatial resolution was calculated by multiplying the resolution along the three orthogonal directions. The measured spatial resolution was not corrected for source size, positron range, or photon acolinearity.

\section{Sensitivity}

The absolute system sensitivity was measured using a droplet of 2 deoxy-2-[18F]fluoro-D-glucose $\left(\left[{ }^{18} \mathrm{~F}\right] \mathrm{FDG}\right)$ solution on a piece of filter paper measuring $1 \mathrm{~mm}^{2}$. The activity on the filter paper was $67 \mathrm{kBq}(1.8 \mu \mathrm{Ci})$ measured in a well-type gamma counter (Wallac Wizard 1480, Perkin Elmer, Shelton, CT). The filter paper was placed between two pieces of 5-mm-thick plastic each measuring $8 \times 8 \mathrm{~mm}$ in size to ensure that all positrons emanating from the source annihilate in the surrounding material. The source was set on a translation stage and positioned midway between the two detectors. Measurements were taken by stepping the source in both the coronal $x$ and $y$ directions with steps of $5 \mathrm{~mm}$. At each location, list-mode data were acquired with a timing window of $20 \mathrm{~ns}$. The data were then histogrammed using an energy window of $150-650 \mathrm{keV}$. Randoms were subtracted from the prompt coincidences to get the number of true coincidences. The recorded counts were corrected for ${ }^{18} \mathrm{~F}$ decay and positron yield, but the attenuation of the plastics surrounding the
${ }^{18} \mathrm{~F}$ source was not compensated, which leads to an estimated $9 \%$ sensitivity loss based on the $0.095 \mathrm{~cm}^{-1}$ attenuation of $511 \mathrm{keV}$ photons in water equivalent material. For each location, the absolute system sensitivity was calculated as the ratio between the recorded true coincidence counting rate and the source activity.

To investigate the sensitivity dependency on the energy threshold, the list-mode data taken at the center of the FOV, which provides the system peak absolute sensitivity, were histogrammed using different energy windows. The system peak absolute sensitivity was measured and analyzed as a function of energy window. The choice of energy window for a PET scanner will affect system sensitivity and the energy window used in typical studies should be determined based on the trade-off between system sensitivity and scatter fraction.

\section{Scatter and Counting-Rate Performance}

Counting-rate performance of the PETbox system was evaluated using the NEMA NU 4-2008 mouse-sized phantom, which is a 70-mm-long and 25-mm diameter solid cylinder made of highdensity polyethylene $(0.96 \mathrm{~g} / \mathrm{cm} 3)$, with a $3.2-\mathrm{mm}$ diameter hole drilled parallel to the central axis at a radial distance of $10 \mathrm{~mm}$. A flexible tube filled with $\left[{ }^{18} \mathrm{~F}\right] \mathrm{FDG}$ solution was inserted into the hole of the phantom. The initial activity was measured to be 13.1 MBq $(355 \mu \mathrm{Ci})$ using a dose calibrator (Atomlab 300; Biodex Medical Systems). The phantom was placed in the system so that the tube was located at the axial center of the FOV to ensure that the dead time experienced by the two detector heads were the same. Data were acquired until the total activity decayed below $10,000 \mathrm{~Bq}$. The timing window was set to $20 \mathrm{~ns}$ during the acquisition. The random coincidences were measured using the delayed window method.

The acquired list-mode data was histogrammed into $2 \mathrm{D}$ sinogram sets with 10-min frame duration using four sets of energy windows (150-650, 250-650, 350-650, and 450-650 keV). Prompts and delayed coincidences were histogrammed separately. Data analysis was performed conforming to the NEMA NU 4-2008 standards. For each prompt sinogram, the profile of each projection angle was shifted so that the peak pixels were aligned with the center pixel of the sinogram. A sum projection was produced by adding up all angular projections in each slice and in each frame. All pixel counts outside of a 14-mm centered band were assumed to be the sum of random and scatter counts. A linear interpolation between the left and right border of the $14 \mathrm{~mm}$ band was used to estimate these non-true counts under the profile peak. Counts above this line were taken as true events. Randoms were estimated from the delayed sonogram.

The scatter fraction was measured using a prompt sinogram with an activity of $210 \mathrm{kBq}(5.67 \mu \mathrm{Ci})$. This low activity frame was chosen to ensure that system dead time and randoms did not affect the measurement. The randoms for this particular frame were found to be less than $0.2 \%$ of the total prompt counts, suggesting they were negligible in this 10 -min measurement. The scatter fraction SF was calculated as

$$
\mathrm{SF}=\frac{R_{\text {scatter }}}{R_{\text {prompt }}}=\frac{R_{\text {prompt }}-R_{\text {true }}}{R_{\text {prompt }}}
$$

where $R_{\text {scatter }}, R_{\text {prompt }}$, and $R_{\text {true }}$ are the number of scatter, prompt, and true events, respectively. 


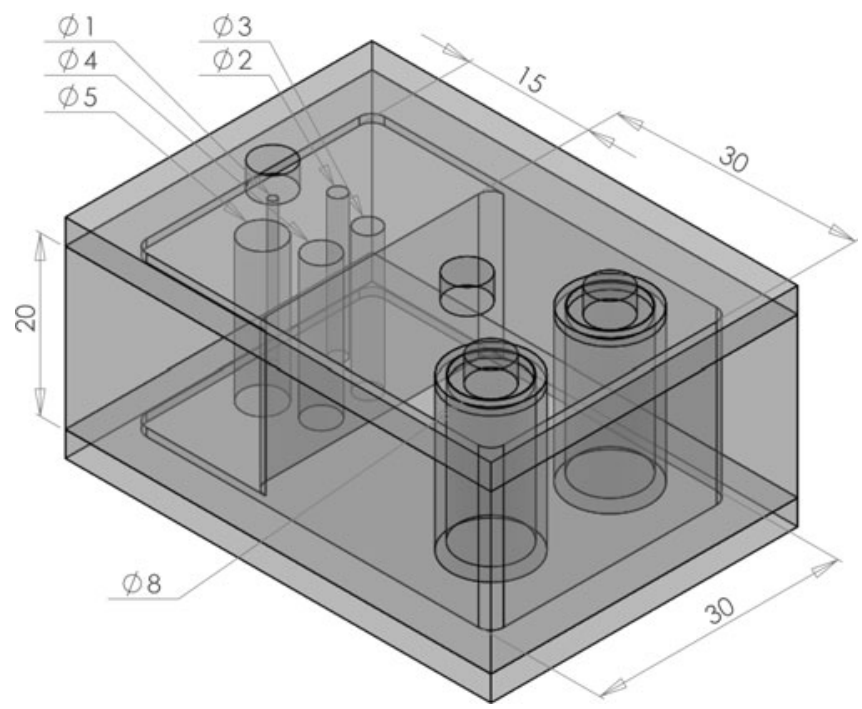

Fig. 2. Drawing of the modified image quality phantom specially designed for the PETbox system. All dimensions are in millimeters.

The noise equivalent count rate (NECR) for each prompt sinogram was calculated using the following formula:

$\mathrm{NECR}=\frac{R_{\text {true }}^{2}}{R_{\text {prompt }}+R_{\text {random }}}=\frac{\left(R_{\text {prompt }}-R_{\text {random }}\right)^{2}(1-\mathrm{SF})^{2}}{R_{\text {prompt }}+R_{\text {random }}}$

where $R_{\text {random }}$ is the number of random events obtained from the delayed sinogram.

\section{Imaging Studies}

Both phantom and in vivo studies were performed to demonstrate the imaging capabilities of the PETbox system. Unless stated, for all the studies shown in the following sections, list-mode data were acquired with a timing window of $20 \mathrm{~ns}$. An energy window of 150-650 keV was applied during list-mode data histogramming. Normalization and random event correction were applied before image reconstruction. Attenuation correction was performed when applicable and was stated in the corresponding text. No scatter correction was performed for any of the studies presented below.

Image Quality Phantom The image quality of the PETbox system was evaluated using an image quality phantom consisting of a main fillable uniform region chamber, two small cold region chambers with one filled with nonradioactive water and the other with air, and a solid acrylic region with five fillable rods of different diameters. The box-shaped image quality phantom, as shown in Fig. 2, is modified from the NEMA NU 4-2008 standard to fit into the PETbox system gantry. Since the PETbox is intended to be used with a low injection dose, the phantom was filled with $1.85 \mathrm{MBq}(50 \mu \mathrm{Ci})\left[{ }^{18} \mathrm{~F}\right] \mathrm{FDG}$ solution in this study. The phantom was placed at the center of the system FOV and scanned for $20 \mathrm{~min}$ using typical settings as stated above.

Normalization, random, and attenuation corrections were applied to the acquired data before image reconstruction, but no scatter correction was applied. The attenuation correction was realized by multiplying the sinograms with an analytically generated attenuation sinogram. A voxelized model of the image quality phantom was created with a cubic voxel size of $0.55 \mathrm{~mm}^{3}$ and a matrix size of $80 \times 80 \times 176$. Each voxel in the phantom was assigned with the attenuation coefficient of water $\left(0.095 \mathrm{~cm}^{-1}\right.$ at $511 \mathrm{keV}$ ) except for the cold region chamber filled with air, which was set to zero. The attenuation sinogram was created by analytically calculating the line integrals of each LOR through the phantom.

Data analysis on the image quality phantom reconstructed images was performed with reference to the NEMA NU 4-2008 standards. A $10 \times 20 \times 6 \mathrm{~mm}^{3}$ volume of interest (VOI) was drawn over the center of the uniform region. The mean value and standard deviation (SD) in this VOI was measured and the percentage SD was calculated to estimate the noise performance of the imaging system. To calculate the recovery coefficient (RC) for each hot rod, a single coronal image was generated by averaging the image slices covering the central 10-mm length of the rods. Circular regions of interest (ROI) were drawn in this image around each rod, with diameters twice the physical diameters of the rods. The RC for each hot rod and the SD of the RCs were obtained as specified in the NEMA NU 4-2008 standard. Although no scatter correction was applied to the acquired dataset, the spillover ratios of the water- and air-filled cold region chamber were calculated as specified in the NEMA NU 4-2008 standard to provide a rough estimation of the scatter effects.

96-Well Plate Study The quantitation capability of the PETbox system was investigated using a specially designed 96-well plate study. Eight wells on the 96-well plate (Greiner Bio-One 655090, USA Scientific, Orlando, FL) were filled with decreasing activities of $\left[{ }^{18}\right.$ F $]$ FDG solutions. As indicated in Fig. 3, the first well was filled with the highest activity of $67.9 \mathrm{kBq}(1.834 \mu \mathrm{Ci})$, and the activity decreased approximately by half for the next well, reaching $629 \mathrm{~Bq}$ $(17.3 \mu \mathrm{Ci})$ for the last one. These activities were calibrated using a well-type gamma counter (Wallac Wizard 1480, Perkin Elmer, Shelton, CT). The 96-well plate was placed midway between the

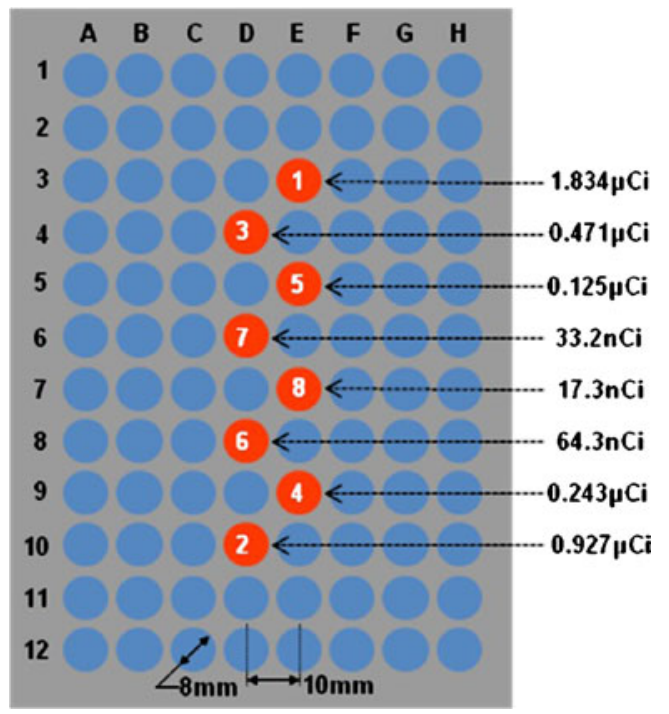

Fig. 3. The pattern of $\left[{ }^{18} \mathrm{~F}\right] \mathrm{FDG}$ solution distribution and calibrated activities in the eight selected wells on the 96-well plate used in the system quantitation study. 
detectors, and data were acquired for $420 \mathrm{~min}$. The recorded list-mode data were histogrammed into 20-min frames, and 3D images were reconstructed for each frame. For each frame, cylindrical ROIs were drawn on the hot wells in the reconstructed images. The diameter of each ROI was set to $9 \mathrm{~mm}$ to cover the well area ( $8 \mathrm{~mm}$ in diameter), and the height of each ROI was set to $9 \mathrm{~mm}$, covering $\pm 4.5 \mathrm{~mm}$ from the central coronal slice. The mean image value for each ROI was calculated and used as a quantitative representative of the activity in the ROI (hot well). These values were compared to the calibrated well activities to reveal the quantitative performance of the system.

In Vivo Mouse Studies A $\left[{ }^{18} \mathrm{~F}\right] \mathrm{FDG}$ study was performed on a healthy mouse to demonstrate the dynamic imaging capability of the PETbox system. The mouse was anesthetized with isofluorane, positioned in the imaging chamber, and placed in the PETbox system. Following a tail injection of $740 \mathrm{kBq}(20 \mu \mathrm{Ci})\left[{ }^{18} \mathrm{~F}\right] \mathrm{FDG}$, the mouse was scanned for $1 \mathrm{~h}$. List-mode data were acquired and sliced into 16 frames, with frame length of $2,3,5,10,10,10,10$, $10,60,60,60,60,300,600,600$, and 1,800 s. For each frame, 3D images were reconstructed using the ML-EM method. No attenuation or scatter correction was applied. ROIs were manually drawn for the bladder, heart, liver, and kidney in the reconstructed images. The mean image value in each ROI was calculated as a representative of activity distribution in that organ.

A static $\left[{ }^{18} \mathrm{~F}\right] \mathrm{FAC}$ study was performed to validate system performance with different probes. $\left[{ }^{18} \mathrm{~F}\right] \mathrm{FAC}$ is a specialized probe targeted for immune function and it is based on deoxyribonucleotide salvage pathway for DNA synthesis [50]. One healthy mouse was injected with $1.85 \mathrm{MBq}(50 \mu \mathrm{Ci})\left[{ }^{18} \mathrm{~F}\right] \mathrm{FAC}$. After an uptake of $100 \mathrm{~min}$, the mouse was scanned for $20 \mathrm{~min}$. 3D images were reconstructed from the acquired list-mode data with no attenuation or scatter correction.

\section{Results}

\section{Detector Performance}

Basic intrinsic performance data of the PETbox detector, including the energy resolution, coincidence timing resolution, and spatial resolution, have already been reported in [41]. The results are summarized here for completeness. The energy resolution for the two BGO arrays ranged from $16.1 \%$ to $38.5 \%$ full width at half maximum (FWHM), with a mean of $20.1 \%$; and from $15.5 \%$ to $42.7 \%$, with a mean of $19.6 \%$, respectively, on the prototype scanner. The coincidence timing resolution of the detector pair was $4.1 \mathrm{~ns}$ FWHM. The intrinsic spatial resolution was measured to range from 1.48 to $2.33 \mathrm{~mm}$ along the coronal $x$ axis (detector long axis) and from 1.55 to $2.39 \mathrm{~mm}$ along the coronal $y$ axis (detector short axis), with an average of $1.78 \mathrm{~mm}$ (see Fig. 1a for the coordinates definition).

\section{Image Spatial Resolution}

Table 1 summarizes the spatial resolution measured with the reconstructed images of the ${ }^{22} \mathrm{Na}$ point source. No significant difference was observed between the two planes at
Table 1. ML-EM reconstructed image spatial resolution

\begin{tabular}{llll}
\hline Plane position & \multicolumn{2}{l}{ Spatial resolution FWHM (mm) } \\
\cline { 2 - 3 } & \multicolumn{2}{l}{ In-plane Coronal } & $\begin{array}{l}\text { Anterior- } \\
\text { Posterior }(z)\end{array}$ \\
\cline { 2 - 3 } & Coronal $x$ & Coronal $y$ & \\
\hline$z=0$ (central coronal plane) & $1.61 \pm 0.12$ & $1.54 \pm 0.22$ & $2.61 \pm 0.47$ \\
$z=6$ mm (offset coronal plane) & $1.55 \pm 0.11$ & $1.43 \pm 0.10$ & $2.69 \pm 0.51$ \\
\hline
\end{tabular}

different $z$ positions. In-plane spatial resolution is rather uniform in both planes, with an isotropic mean value of $1.53 \mathrm{~mm}$ FWHM. Anterior-posterior plane spatial resolution has a mean value of $2.65 \mathrm{~mm}$, and the degradation is caused by incomplete LOR collection due to the system geometry. Fig. 4 shows the volumetric resolution at different locations in the two coronal planes. It ranges from 3.99 to $12.82 \mathrm{~mm}^{3}$ for the central coronal plane and from 4.08 to $13.80 \mathrm{~mm}^{3}$ for the offset coronal plane. The volumetric resolution is below $8 \mathrm{~mm}^{3}$ at most locations except for near the edge of the FOV. For comparison, we also plotted the image spatial resolution in the radial, tangential, and axial directions measured in the central coronal plane in compliance with the NEMA NU 4-2008 standard, as shown in Fig. 5. The radial and axial resolutions are all below $2 \mathrm{~mm}$ FWHM, while the tangential resolutions are worse. However, the volumetric resolutions are all below $8 \mathrm{~mm}^{3}$, which is within the design specifications of the system.

\section{Sensitivity}

Fig. 6a plots the measured absolute system sensitivity as a function of the source location along both the coronal $x$ and $y$ directions. The peak absolute sensitivity of the system at the center of the FOV is measured to be $3.99 \%$, and the sensitivity drops for positions away from the center. It is worthwhile to note that the measured sensitivity was not corrected for source self-attenuation (corresponding to approximately $9 \%$ sensitivity loss).

Fig. $6 \mathrm{~b}$ and $\mathrm{c}$ shows the measured system peak absolute sensitivity as a function of energy windows. In Fig. 6b, the upper energy threshold (ULD) is fixed at $650 \mathrm{keV}$ and the lower energy threshold (LLD) changes from 100 to $250 \mathrm{keV}$; while in Fig. 6c, the LLD is fixed at $150 \mathrm{keV}$ and the ULD changes from 650 to $750 \mathrm{keV}$. It is evident that the sensitivity has a strong dependency on the lower energy threshold, dropping from $4.6 \%$ at $100 \mathrm{keV}$ LLD to $2.8 \%$ at $250 \mathrm{keV}$ LLD. The upper energy threshold, however, has little effect on the sensitivity, which increases only slightly as the ULD goes up from 650 to $750 \mathrm{keV}$.

\section{Scatter and Counting-Rate Performance}

Table 2 lists the measured scatter fraction with different energy windows. These measured scatter fractions are higher compared with those previously reported for other systems 

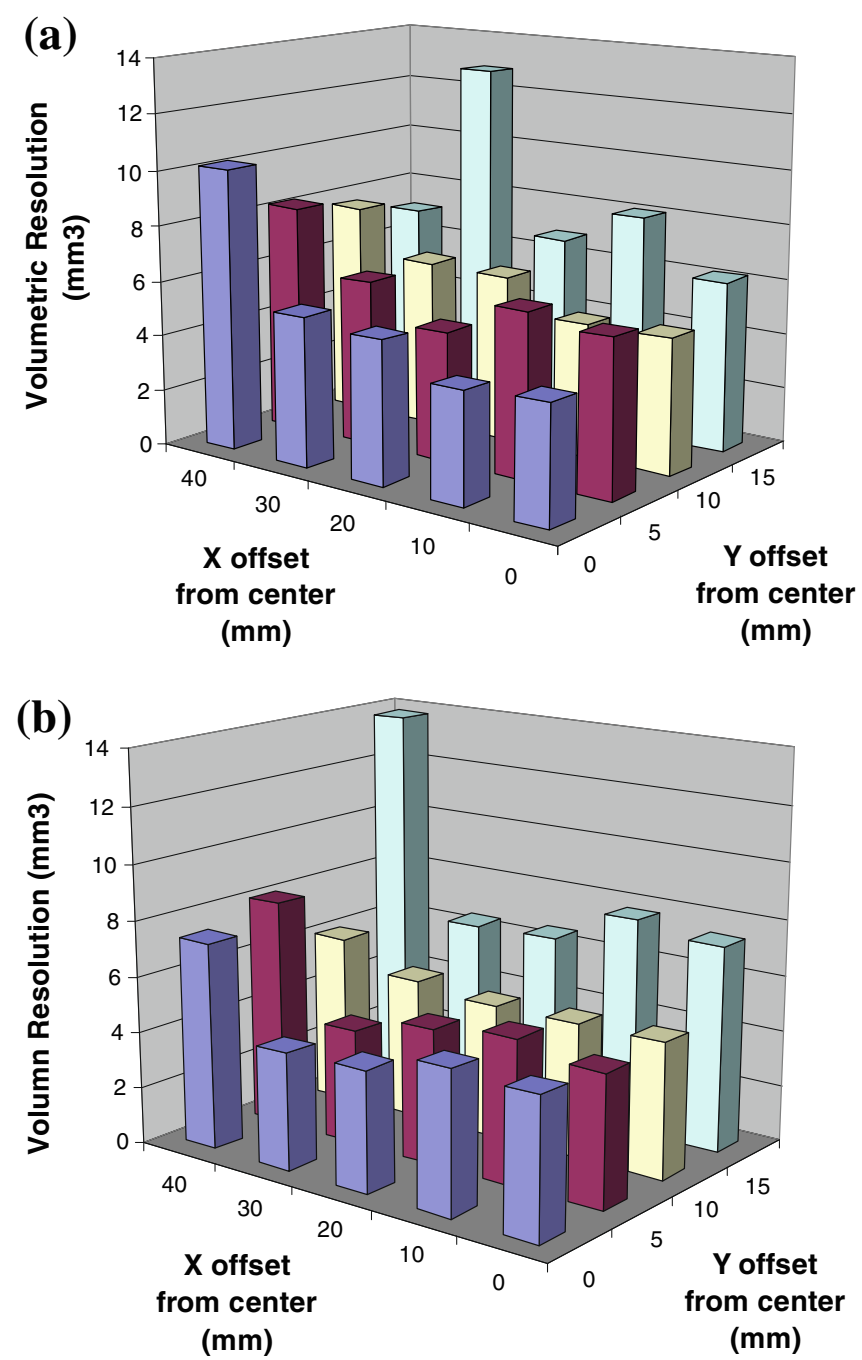

Fig. 4. Volumetric spatial resolutions of the PETbox system measured with a point source at different locations in a the central coronal plane and $\mathbf{b}$ the $6 \mathrm{~mm}$ offset coronal plane. Images were reconstructed using the ML-EM method described in the text.

[23, 51], especially with lower energy thresholds. This is believed to be caused in part by the materials associated with the system gantry and the closed geometry of the PETbox system $(\sim 43.6 \%$ solid angle). Those materials include the aluminum-based detector brackets as well as a special plastic guide that sits on top of the bottom detector and serves as the guiding rail for the mouse chamber (see Fig. 1a).

Fig. 7 shows the measured prompt and random rates and the calculated NECR curve for the mouse-sized phantom with two energy windows. The prompt rates reach peak values at around $3.7 \mathrm{MBq}(100 \mu \mathrm{Ci})$ total activity with both energy windows. With this activity, the random events account for approximately $3 \%$ of the total prompt counts. The PETbox detector pair was previously measured to have a timing resolution of $4.1 \mathrm{~ns}$ FWHM [41], but since the random rate is very low, a large timing window can be used to increase the system sensitivity, as was done in all the measurements presented in this manuscript. The peak NECR is calculated as $20 \mathrm{kcps}$ for the $150-650 \mathrm{keV}$ and $18.2 \mathrm{kcps}$ for the 250-650 keV energy window, both achieved at a total activity of $3.2 \mathrm{MBq}(86.8 \mu \mathrm{Ci})$ in the phantom, suggesting that the NECR of the PETbox system is mainly limited by the counting rate capability of the BGO detector. Because the scatter fraction for mouse imaging is relatively small, a wide open energy window maximizes system sensitivity without significantly compromising imaging results. For optimized mouse imaging, an energy window of $150-650 \mathrm{keV}$ and a timing window of 20 ns were chosen for the PETbox system.

\section{Imaging Studies}

Image Quality Phantom Fig. 8 illustrates a reconstructed coronal slice of the image quality phantom, showing the five hot rods on the top, the uniform region in the middle, and the two cold chambers in the bottom. Uniformity measurement was performed on the uniform region. The mean value and standard deviation (SD) in the $10 \times 20 \times 6 \mathrm{~mm}^{3}$ VOI drawn over the center of the uniform region was measured to be 921.4 and 90.8 , respectively, and the percentage SD was

(a)

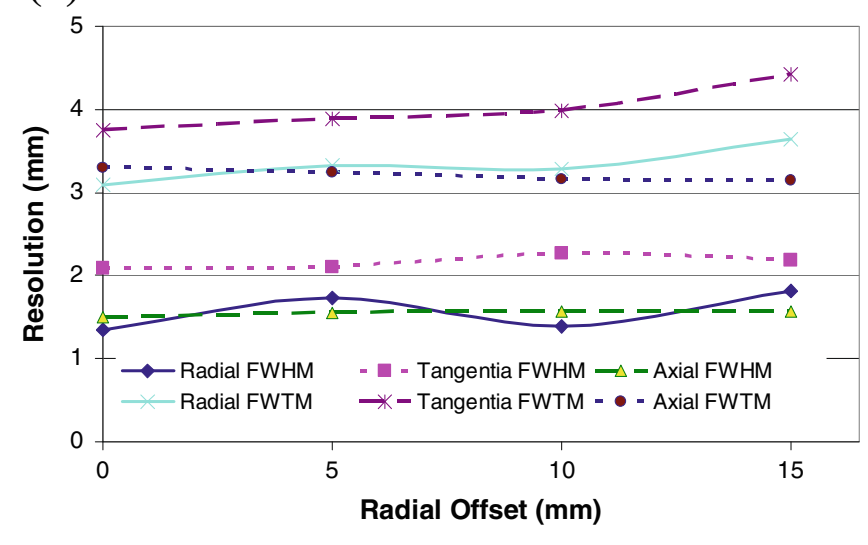

(b)

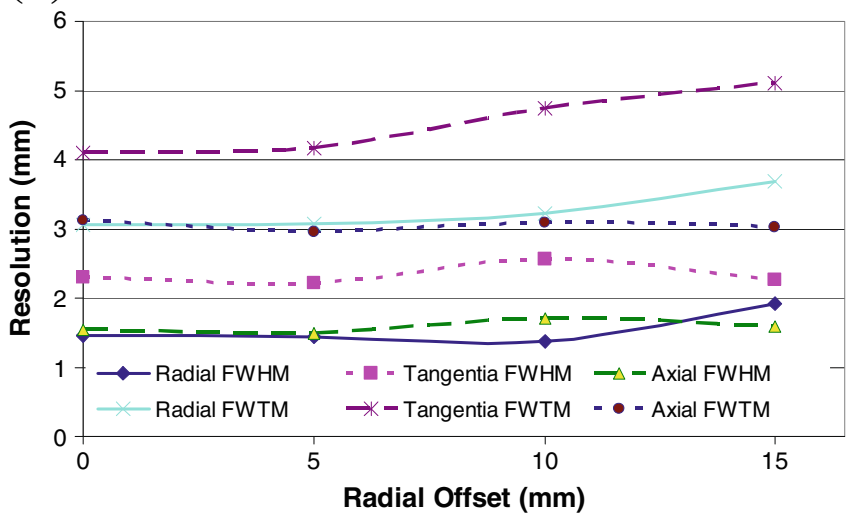

Fig. 5. ML-EM reconstructed image resolution of the PETbox system plotted per NEMA NU 4-2008 protocol, showing the FWHM and FWTM of the radial, tangential, and axial image resolutions at $\mathbf{a}$ axial center of the FOV and $\mathbf{b} 20 \mathrm{~mm}$ from the axial center towards the axial edge of the FOV. 
a

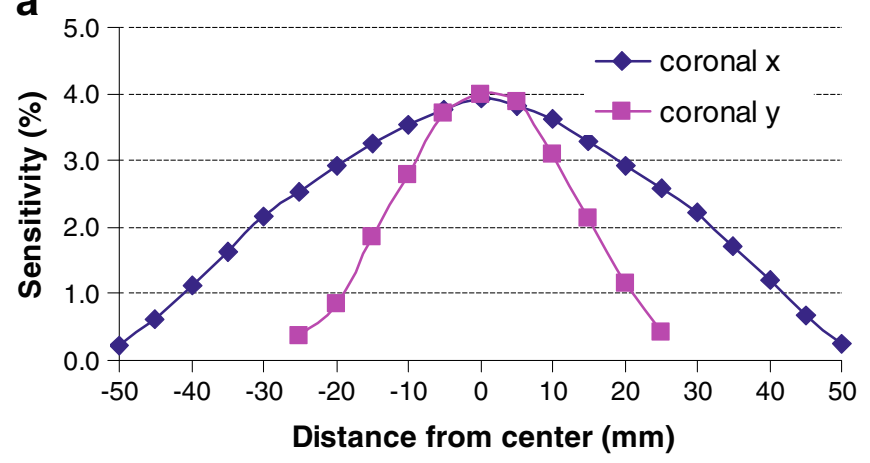

b
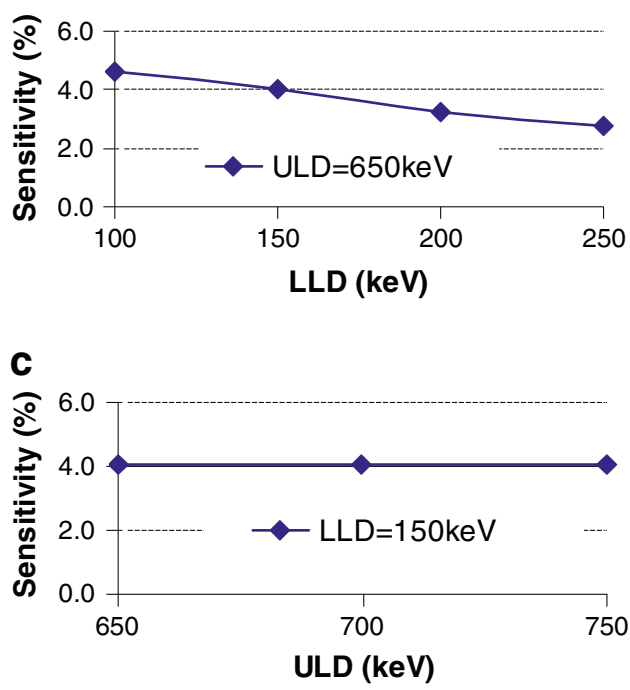

Fig. 6. a Measured absolute system sensitivity as a function of distance from the center in both the coronal $x$ and $y$ directions. The energy window was 150-650 keV for this measurement. The peak absolute sensitivity as a function of energy threshold is shown in $\mathbf{b}$ by changing the lower energy threshold (LLD) and in $\mathbf{c}$ by changing the upper energy threshold (ULD).

9.9. The percentage SD in this uniform region provides a measure to estimate the uniformity of the imaging system. However, because of the Poisson distribution of the signal, the actual value is also directly related to the injection dose, scan time, and system sensitivity. Taking those into consideration, the percentage SD obtained in our measurement is comparable to that previously reported for a commercial full-ring system with a sensitivity of $6.72 \%$ measured at $350-625 \mathrm{keV}$. For that system, a percentage SD of 5.29 was obtained from a 20-min scan with $5.1 \mathrm{MBq}$

Table 2. Scatter fraction for the mouse-sized phantom $\left(34 \mathrm{~cm}^{3}\right)$

\begin{tabular}{lc}
\hline Energy Window & Scatter Fraction \\
\hline $150-650 \mathrm{keV}$ & $21.3 \%$ \\
$250-650 \mathrm{keV}$ & $14.3 \%$ \\
$350-650 \mathrm{keV}$ & $9.2 \%$ \\
$450-650 \mathrm{keV}$ & $5.2 \%$ \\
\hline
\end{tabular}
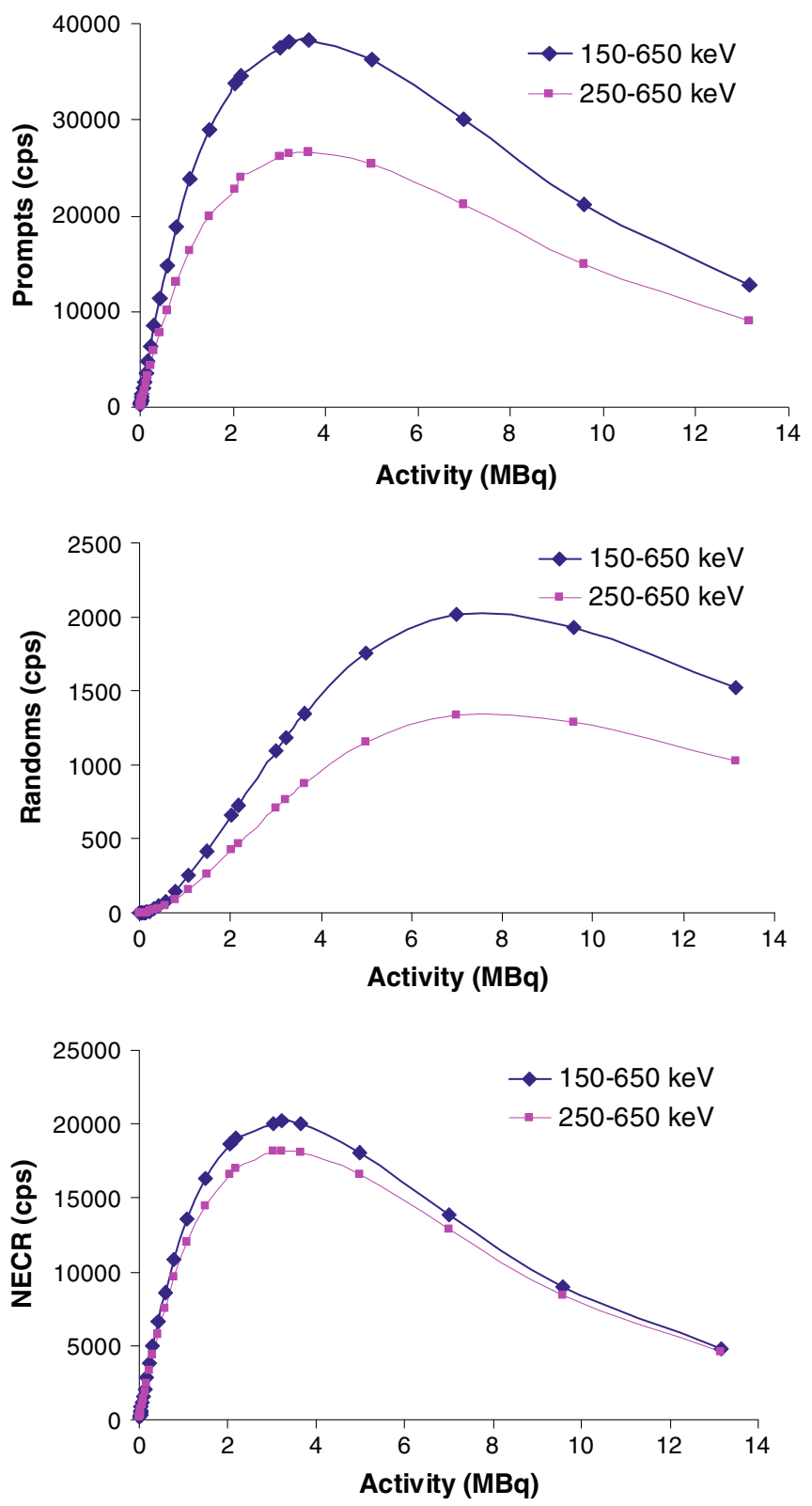

Fig. 7. Measured prompt, random, and NEC rates as a function of total activity in the mouse-sized phantom. The timing window was $20 \mathrm{~ns}$.

$(137.8 \mu \mathrm{Ci})$ total activity in the phantom [23], resulting in approximately 3.5 times more true events for that measurement than the events obtained here.

The calculated recovery coefficients and standard deviations of the five hot rods were summarized in Table 3. It is worthwhile to note that no scatter correction was applied for the acquired dataset, resulting in a higher mean image value in the uniform region than normal, which in turn leads to an underestimation of the recovery coefficients for the system [23]. The spillover ratios of the water- and air-filled cold region chamber were calculated to be $14.8 \%$ and $3.3 \%$, respectively. Again, these numbers were obtained without scatter correction. 


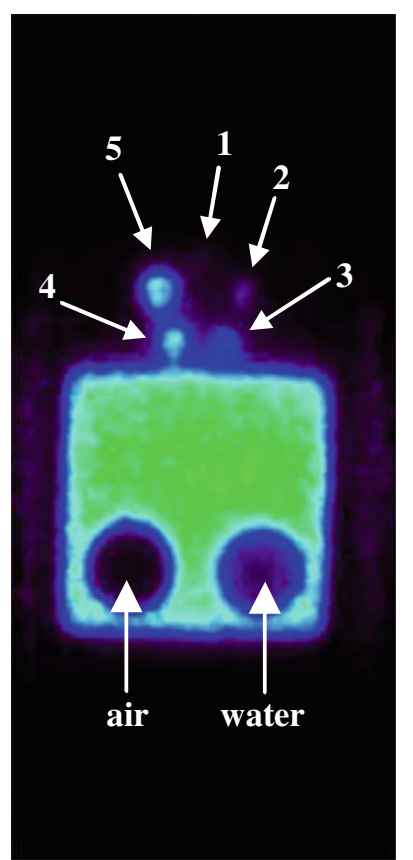

Fig. 8. A reconstructed coronal slice of the image quality phantom. The phantom was filled with $1.85 \mathrm{MBq}(50 \mu \mathrm{Ci})$ $\left[{ }^{18} \mathrm{~F}\right]$ FDG solution and scanned for $20 \mathrm{~min}$.

96-Well Plate Study Fig. 9a and b shows the reconstructed central coronal slice of the first and last (the 21st) 20-min frames, respectively, with the hottest and weakest well activities indicated for each frames. The mean image value of each ROI drawn on the hot wells was calculated and plotted against the calibrated well activities. As shown in Fig. 9c, good linearity was observed between the mean ROI image values and the activities. The system also demonstrates a wide quantitation range, from 67.9 $(1.834 \mu \mathrm{Ci})$ to $51.4 \mathrm{~Bq}(1.39 \mathrm{nCi})$ for the acquisition presented here.

In Vivo Mouse Studies Fig. 10 shows selected reconstructed coronal slices from eight frames corresponding to the time period: (a) 0-2 s, (b) 2-5 s, (c) 5-10 s, (d) 30-40 s, (e) 1-2 min, (f) 4-5 min, (g) 10-20 min, and (h) 30-60 min following the injection in the dynamic $\left[{ }^{18} \mathrm{~F}\right] \mathrm{FDG}$ study. The reconstructed images show the tail injection in early frames and the activity building up in kidney, liver, bladder, and heart in later frames. The measured time-activity curve in the bladder, heart, liver, and kidney are plotted in Fig. 11, which clearly reveals the trend of activity distribution in these organs as time progresses. Fig. 12 shows a recon-

Table 3. Report for recovery coefficient tests

\begin{tabular}{lccccc}
\hline Rod Diameter & $1 \mathrm{~mm}$ & $2 \mathrm{~mm}$ & $3 \mathrm{~mm}$ & $4 \mathrm{~mm}$ & $5 \mathrm{~mm}$ \\
\hline RC & 0.08 & 0.24 & 0.51 & 0.72 & 0.72 \\
$\% \mathrm{SD}$ & 13.5 & 11.5 & 17.3 & 18.3 & 18.0 \\
\hline
\end{tabular}

structed coronal slice in the static $\left[{ }^{18} \mathrm{~F}\right] \mathrm{FAC}$ study. The $\left[{ }^{18} \mathrm{~F}\right]$ FAC probe is targeted for the immune system, and it allows visualization of thymus and spleen in mice, which are clearly shown in Fig. 12.

\section{Discussion and Conclusion}

The PETbox system was designed as low cost option for pharmacokinetic and pharmacodynamic studies in mice. Compared with commercially available small animal PET scanners, the bench top configuration of this system greatly reduces the cost as well as the space needed for researchers to access PET imaging technology. While a very high performance scanner was not the goal of this work, the key performance metrics for PETbox were chosen to satisfy typical resolution and sensitivity requirements for routine biodistribution and organ uptake quantification studies [31, 33]. The evaluation presented in this manuscript demonstrates that the prototype system meets the intended design specifications. The tomograph was also designed with an integrated mouse handling system that in addition to anesthesia and temperature control provides reproducible positioning and locates subjects within the optimal location in the FOV [52]. The measured spatial resolution within this effective imaging volume is $1.53 \mathrm{~mm}$ FWHM in the coronal directions and $2.65 \mathrm{~mm}$ in the AP direction, with minimal variations. For this region, the volumetric spatial resolution averages $6 \mathrm{~mm}^{3}$ and is not dramatically different than the spatial resolution of many other dedicated preclinical imaging instruments.

Many successive generations of preclinical imaging system have been developed since some of the early work in which the optimal injected dose for mouse studies was established [53]. System design, sensitivities, and count rate capabilities have changed dramatically since then. The design of the PETbox took into renewed consideration a combination of subject centered factors, including the need to perform longitudinal studies in mice bearing tumors, where perhaps ten or more successive studies are performed in a period of a few weeks on the same animal. In addition, preclinical PET as a high-sensitivity imaging modality is often required to image very weak signals such as those from gene expression imaging [54] or from imaging immune system responses [50]. Based on these considerations, the PETbox was designed with the task to optimize imaging with routine injections of $1.85 \mathrm{MBq}(50 \mu \mathrm{Ci})$. At this level of injected activity, serial protocols of ten studies give rise to a dose of $25 \mathrm{cGy}$ to background soft tissues and $55 \mathrm{cGy}$ to soft tissue tumors with an average SUV of 2 [38]. While this dose level still is not trivial, it is significantly less (approximately four times less) than the dose from the standard injections of $7.4 \mathrm{MBq}(200 \mu \mathrm{Ci})$ used today. An added benefit from this low injected activity is a reduced exposure to the scanner operator.

The use of the PETbox with low levels of administered dose was achieved by employing a compact system 
a

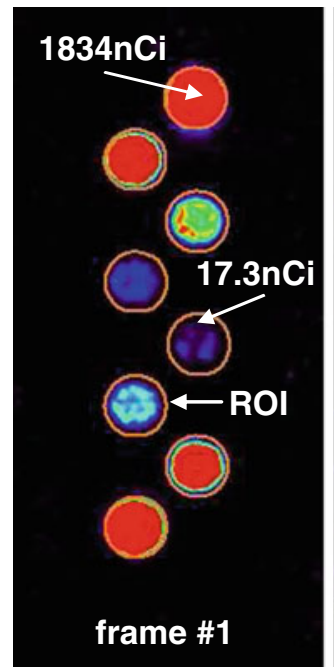

b

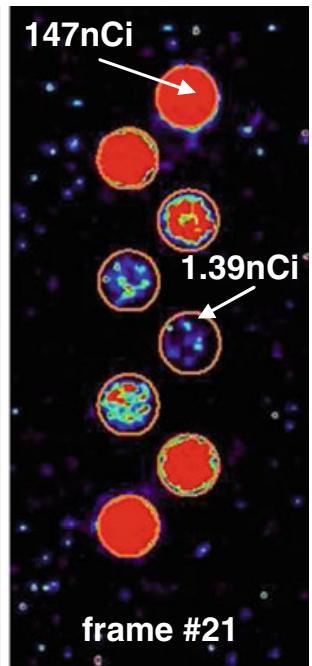

C

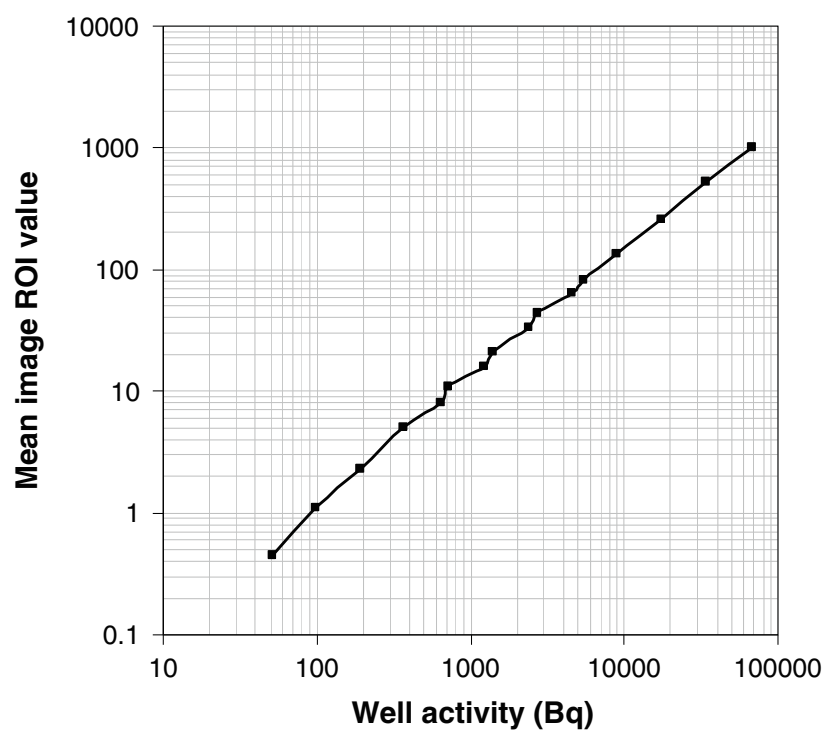

Fig. 9. Reconstructed central coronal slice of the $\mathbf{a}$ first frame and $\mathbf{b}$ last frame. Different color scales are used for the two images for better visualization of the weakest activity in each image. ROI regions for each hot well are also shown on the images. c The mean image ROI values are plotted as a function of calibrated well activities.
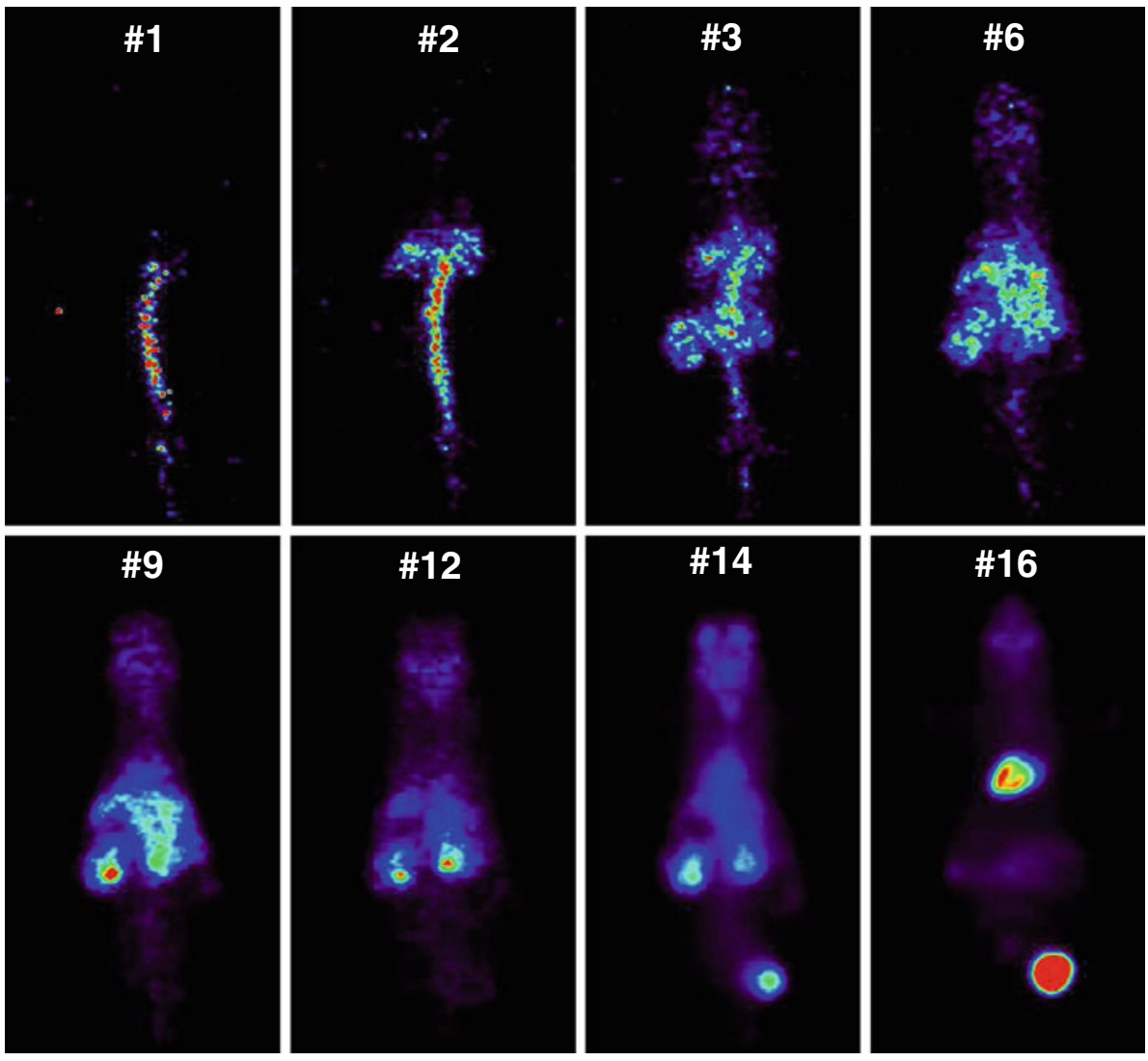

Fig. 10. Selected frames from the $1-\mathrm{h}$ dynamic scan of a mouse following a tail injection of $740 \mathrm{kBq}(20 \mu \mathrm{Ci})\left[{ }^{18} \mathrm{~F}\right] \mathrm{FDG}$. Different coronal slices are shown for each frame to better reveal the activity distribution for different time periods. 

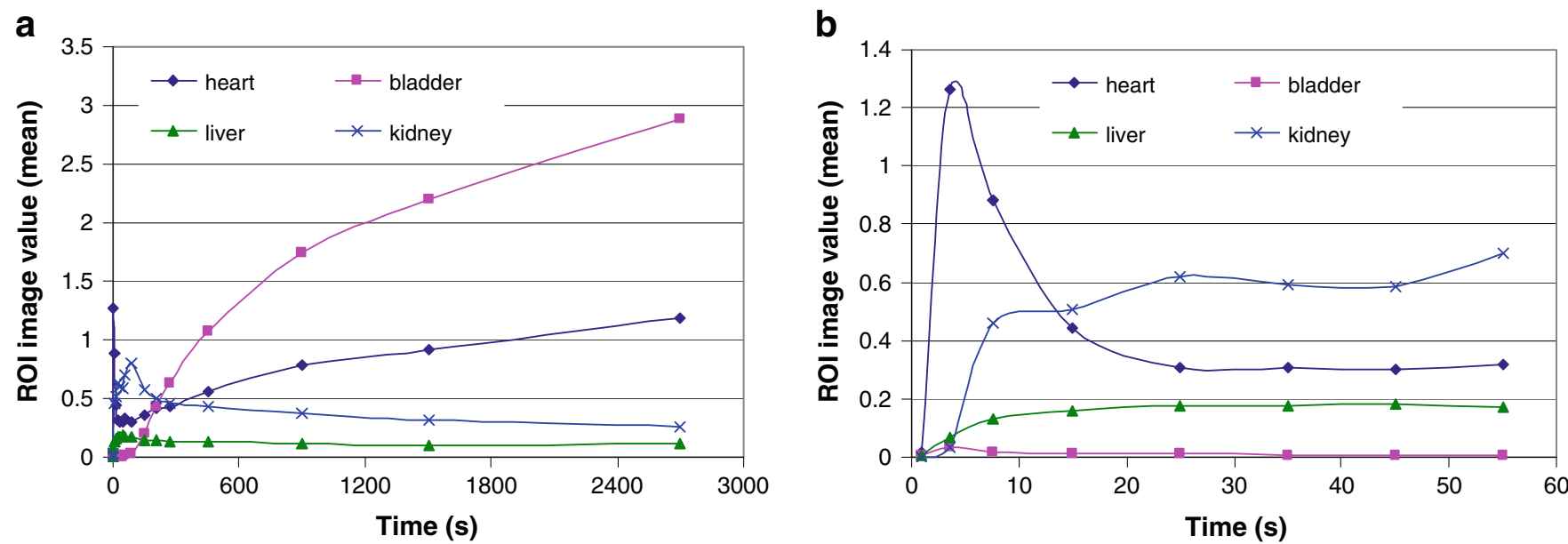

Fig. 11. Time activity curves in major organs of a mouse during the $1 \mathrm{~h}$ dynamic $\left[{ }^{18} \mathrm{~F}\right] \mathrm{FDG}$ scan. a Organ uptake for the $1-\mathrm{h}$ period. b Close-up for the first $60 \mathrm{~s}$ following injection.

geometry optimized for imaging mice as well as by using a BGO scintillator which has very high stopping power for $511 \mathrm{keV}$ photons and very low intrinsic radioactivity. Our simulation shows that for the PETbox geometry, even at a lower LLD of $150 \mathrm{keV}$, BGO has less crystal scatter and higher photoelectrical fraction compared with LSO at $250 \mathrm{keV}$ LLD [39], suggesting that a wide open energy window can be used for the PETbox system to collect events. The peak absolute sensitivity was measured to be $3.99 \%$ with a $150-650 \mathrm{keV}$ energy window, which agrees very well with the simulation. The measured NECR curve shows a favorable system operating range below a total

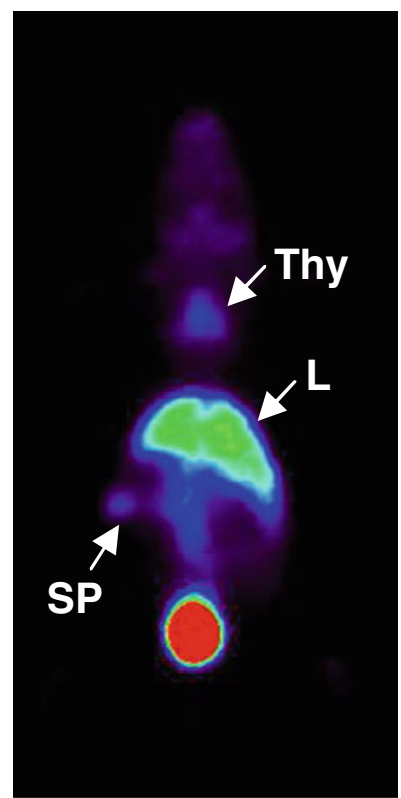

Fig. 12. A reconstructed coronal slice showing the uptake of $\left[{ }^{18} \mathrm{~F}\right] \mathrm{FAC}$ in spleen and thymus in a healthy mouse during a 20 -min scan. The injection was $1.85 \mathrm{MBq}(50 \mu \mathrm{Ci})$ and the uptake time was $100 \mathrm{~min}$. $L$ liver, $S P$ spleen, Thy thymus. activity of $3.7 \mathrm{MBq}(100 \mu \mathrm{Ci})$. Both the phantom and in vivo imaging studies presented in this manuscript also demonstrate the utility of the system at low activity levels. A tradeoff though inherent in this approach is that the system does not operate optimally when there are more than $3.7 \mathrm{MBq}$ $(100 \mu \mathrm{Ci})$ activity in the FOV. While as we have demonstrated here, this does not pose a significant problem for routine biodistribution studies, it could pose a challenge for long dynamic imaging of short-lived isotopes, such as those labeled with ${ }^{11} \mathrm{C}$.

Due to the dual-head geometry, PETbox provides a limited angle tomography along the central AP view. Image reconstruction for such geometry depends on the accurate system modeling [55]. The PETbox system P-matrix was generated using GATE simulation and was incorporated into the ML-EM reconstruction method. As a result, reasonably good quantitative results were obtained by the system, as demonstrated by the initial 96-well plate study. Our simulation also shows good quantification accuracy for most large organs and suggests that the quantification capability of the system is mainly limited by the spatial resolution [39]. This is in line with a previous study by He and Frey [56] where a dual-headed system incorporating a full system response provided accurate quantitative results. A full investigation of the quantification accuracy of the PETbox system still needs to be performed in future work with phantom and in vivo imaging.

Finally, it is worthwhile to note that the performance of a small animal PET scanner is conventionally characterized using the NEMA NU 4-2008 Small Animal PET Standard. However, since PETbox has a dual-head geometry instead of a cylindrical geometry of typical full-ring small animal PET scanners, the NEMA NU 4-2008 standards are not always appropriate for the evaluation of such a system. This was also reflected in some other studies performed on a clinical PEM system with similar geometry $[57,58]$. In the work presented here, the NEMA NU 4-2008 standards were used as general guidelines and the actual procedures were 
modified whenever necessary to provide more meaningful results for the proposed system.

Acknowledgments. The authors thank Dirk Williams and Darin Williams for building all the mechanical components for the system. We thank Lily Wu and Caius Radu for assistance with configuring the in vivo experiments and Waldemar Ladno for performing the in vivo studies. This work was supported by the National Institutes of Health under grant no. R24 CA92865 and the Department of Energy under contract no. DE-FC03-02ER63420. Fernando R. Rannou was funded by FONDECYT grant no. 1080465 Chile.

Conflict of Interest Disclosure. A technology similar to the PETbox presented in this manuscript is currently being commercialized by Sofie Bioscience, Inc., Culver City, CA, USA. Nam T. Vu is currently an employee of Sofie Bioscience, Inc., Robert W. Silverman and Richard Taschereau are currently consultants for Sofie Bioscience, Inc., and David B. Stout and Arion F. Chatziioannou are shareholders of Sofie Bioscience, Inc.

Open Access. This article is distributed under the terms of the Creative Commons Attribution Non-Commercial License which permits any noncommercial use, distribution, and reproduction in any medium, provided the original author(s) and source are credited.

\section{References}

1. Phelps ME (2000) Positron emission tomography provides molecular imaging of biological processes. Proc Natl Acad Sci 97(16):9226-9233

2. Gambhir SS (2002) Molecular imaging of cancer with positron emission tomography. Nat Rev Cancer 2(9):683-693

3. Bloomfield PM et al (1995) The design and physical characteristics of a small animal positron emission tomograph. Phys Med Biol 40(6):1105-1126

4. Lecomte $\mathrm{R}$ et al (1996) Initial results from the Sherbrooke avalanche photodiode positron tomograph. IEEE Trans Nucl Sci 43(3):1952-1957

5. Cherry SR et al (1997) MicroPET: a high resolution PET scanner for imaging small animals. IEEE Trans Nucl Sci 44(3):1161-1166

6. Thompson CJ et al. (1998) ANIPET: a versatile PET scanner for imaging small animals. In Proc IEEE Nuclear Science Symp Conf Rec

7. Jeavons AP, Chandler RA, Dettmar CAR (1999) A 3D HIDAC-PET camera with sub-millimetre resolution for imaging small animals. IEEE Trans Nucl Sci 46(3):468-473

8. Di Domenico G et al (2003) YAP-(S)PET small animal scanner: quantitative results. IEEE Trans Nucl Sci 50(5):1351-1356

9. Surti $\mathrm{S}$ et al (2003) Design evaluation of A-PET: a high sensitivity animal PET camera. IEEE Trans Nucl Sci 50(5):1357-1363

10. Seidel J, Vaquero JJ, Green MV (2003) Resolution uniformity and sensitivity of the NIH ATLAS small animal PET scanner: comparison to simulated LSO scanners without depth-of-interaction capability. IEEE Trans Nucl Sci 50(5):1347-1350

11. Tai YC et al (2003) MicroPET II: design, development and initial performance of an improved microPET scanner for small-animal imaging. Phys Med Biol 48(11):1519-1537

12. Correia JA et al (2004) Performance evaluation of MMP-II: a secondgeneration small animal PET. IEEE Trans Nucl Sci 51(1):21-26

13. Rouze NC et al (2004) Design of a small animal PET imaging system with 1 microliter volume resolution. IEEE Trans Nucl Sci 51(3):757-763

14. Miyaoka RS et al (2005) Development of a single detector ring micro crystal element scanner (MiCES): QuickPET II. Mol Imag 4:117-127

15. Ziemons $\mathrm{K}$ et al (2005) The ClearPET(TM) project: development of a 2nd generation high-performance small animal PET scanner. Nucl Instrum Methods Phys Res A 537(1-2):307-311

16. $\mathrm{Li} \mathrm{H}$ et al (2007) The engineering and initial results of a transformable low-cost high-resolution PET camera. IEEE Trans Nucl Sci 54 (5): $1583-1588$

17. Bergeron $\mathrm{M}$ et al (2009) Performance evaluation of the LabPET APDbased digital PET scanner. IEEE Trans Nucl Sci 56(1):10-16

18. Schafers KP et al (2005) Performance evaluation of the 32-Module quadHIDAC small-animal PET Scanner. J Nucl Med 46(6):996-1004

19. Tai YC et al (2005) Performance evaluation of the microPET Focus: a third-generation microPET scanner dedicated to animal imaging. J Nucl Med 46(3):455-463
20. Wang Y et al (2006) Performance evaluation of the GE healthcare eXplore VISTA dual-ring small-animal PET scanner. J Nucl Med 47 (11):1891-1900

21. Huisman $M$ et al (2007) Performance evaluation of the Philips MOSAIC small animal PET scanner. Eur J Nucl Med Mol Imaging 34(4):532-540

22. de Jong HWAM et al (2007) Performance evaluation of the ECAT HRRT: an LSO-LYSO double layer high resolution, high sensitivity scanner. Phys Med Biol 52(5):1505-1526

23. Bao Q et al (2009) Performance evaluation of the inveon dedicated PET preclinical tomograph based on the NEMA NU-4 standards. J Nucl Med 50:401-408

24. Del Guerra A et al (1998) YAP-PET: first results of a small animal positron emission tomograph based on YAP:Ce finger crystals. IEEE Trans Nucl Sci 45(6):3105-3108

25. Siegel $\mathrm{S}$ et al (1999) Initial results from a PET/planar small animal imaging system. IEEE Trans Nucl Sci 46(3):571-575

26. Weber S et al (1999) Evaluation of the TierPET system. IEEE Trans Nucl Sci 46(4):1177-1183

27. Lage E et al (2009) Design and performance evaluation of a coplanar multimodality scanner for rodent imaging. Phys Med Biol 18:54275441

28. Stickel JR, Cherry SR (2005) High-resolution PET detector design: modelling components of intrinsic spatial resolution. Phys Med Biol 50 (2):179-195

29. Tai YC, Laforest R (2005) Instrumentation aspects of animal PET. Annu Rev Biomed Eng 7(1):255-285

30. Lewellen TK (2008) Recent developments in PET detector technology. Phy Med Biol 17:R287-R317

31. Palm S et al (2003) Pharmacokinetics and biodistribution of 86Ytrastuzumab for $90 \mathrm{Y}$ dosimetry in an ovarian carcinoma model: correlative MicroPET and MRI. J Nucl Med 44(7):1148-1155

32. Malakoff D (2000) SUPPLIERS: the rise of the mouse, biomedicine's model mammal. Science 288(5464):248-253

33. Wester $\mathrm{H}$ et al (2003) PET imaging of somatostatin receptors: design, synthesis and preclinical evaluation of a novel 18F-labelled, carbohydrated analogue of octreotide. Eur J Nucl Med Mol Imaging 30(1):117122

34. Knoess $\mathrm{C}$ et al (2003) Performance evaluation of the microPET R4 PET scanner for rodents. Eur J Nucl Med Mol Imaging 30(5):737747

35. Gagnon MKJ et al (2009) High-throughput in vivo screening of targeted molecular imaging agents. Proc Natl Acad Sci 106 (42):17904-17909

36. Stout $\mathrm{D}$ et al (2005) Small animal imaging center design: the facility at the UCLA Crump Institute for Molecular Imaging. Mol Imag Biol 7 (6):393-402

37. Funk T, Sun M, Hasegawa BH (2004) Radiation dose estimate in small animal SPECT and PET. Med Phys 31(9):2680-2686

38. Taschereau R, Chatziioannou AF (2007) Monte Carlo simulations of absorbed dose in a mouse phantom from 18-fluorine compounds. Med Phys 34(3): 1026-1036

39. Bao Q et al. (2010) Design of a low cost benchtop preclinical PET system (PETbox) based on Monte Carlo simulations. Submitted to Med Phys

40. Zhang $\mathrm{H}$ et al. (2009) Performance evaluation of PETbox: a low cost bench top PET scanner dedicated to high throughput preclinical imaging. In Proc IEEE Nuclear Science Symp Conf Rec

41. Zhang $\mathrm{H}$ et al (2009) Performance characteristics of BGO detectors for a low cost preclinical PET scanner. IEEE Trans Nucl Sci 57(3):10381044

42. Zhang $\mathrm{H}$ et al. (2010) An FPGA-based data acquisition system for PETbox: a low cost preclinical PET scanner. Submitted to IEEE Trans Nucl Sci

43. Performance Measurements of Small Animal Positron Emission Tomographs, 2008, National Electrical Manufacturers Association, NEMA Standards Publication NU 4-2008

44. Murthy $\mathrm{K}$ et al (2000) Positron emission mammographic instrument: initial results. Radiology 215(1):280-285

45. Kao CM et al (2009) A high-sensitivity small-animal PET scanner: development and initial performance measurements. IEEE Trans Nucl Sci 56(5):2678-2688

46. Bao Q, Chatziioannou AF (2007) GATE simulation of a BGO based high sensitivity small animal PET scanner. In Proc Noninvasive 
Functional Source Imaging of the Brain and Heart \& Inter Conf Functional Biomedical Imaging

47. Goertzen AL, Suk JY, Thompson CJ (2007) Imaging of weak-source distributions in LSO-based small-animal PET scanners. J Nucl Med 48 (10): 1692-1698

48. Bao Q et al (2009) Image reconstruction for PETbox, a benchtop preclinical PET tomograph. In Proc IEEE Nuclear Science Symp Conf Rec

49. Taschereau R et al. (2009) Video image based attenuation correction for PETbox, a preclinical PET tomograph. In Proc IEEE Nuclear Science Symp Conf Rec

50. Radu CG et al (2008) Molecular imaging of lymphoid organs and immune activation by positron emission tomography with a new [18F]labeled 2[prime]-deoxycytidine analog. Nat Med 14(7):783-788

51. Yang Y et al (2004) Optimization and performance evaluation of the microPET II scanner for in vivo small-animal imaging. Phys Med Biol 49(12):2527-2545

52. Chow PL et al (2006) A method of image registration for small animal, multi-modality imaging. Phy Med Biol 51(2):379
53. Chatziioannou AF et al (1999) Performance evaluation of microPET: a high-resolution lutetium oxyorthosilicate PET scanner for animal imaging. J Nucl Med 40(7):1164-1175

54. Gambhir SS (2000) Imaging transgene expression with radionuclide imaging technologies. Neoplasia 2:118-138

55. Moses WW, Qi J (2003) Fundamental limits of positron emission mammography. Nucl Instrum Methods Phys Res A 497(1):8289

56. He B, Frey EC (2006) Comparison of conventional, model-based quantitative planar, and quantitative SPECT image processing methods for organ activity estimation using In-111 agents. Phys Med Biol 16:3967-3981

57. MacDonald L et al (2009) Clinical imaging characteristics of the positron emission mammography camera: PEM Flex Solo II. J Nucl Med 50(10):1666-1675

58. Luo W, Anashkin E, Matthews CG (2010) Performance evaluation of a PEM scanner using the NEMA NU 4-2008 small animal PET standards. IEEE Trans Nucl Sci 57(1):94-103 